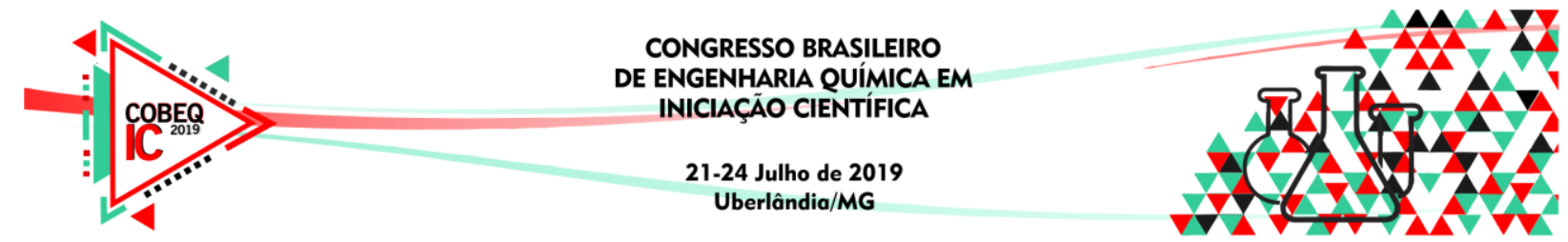

\title{
DESENVOLVIMENTO DE METODOLOGIAS ANALÍTICAS PARA QUANTIFICAÇÃO DE METAIS EM ALIMENTOS: O CASO DO NÍQUEL
}

\author{
N.C. ARAUJO ${ }^{1}$, D. G. FILHO ${ }^{1}$ e D. DE SOUZA ${ }^{1}$ \\ ${ }^{1}$ Universidade Federal de Uberlândia, Campus de Patos de Minas, \\ E-mail para contato: natalia29_11@hotmail.com; djenaine@ufu.br
}

\begin{abstract}
RESUMO - A exposição a altas concentrações de metais pode causar efeitos tóxicos ao ser humano, sendo assim é de fundamental importância desenvolver metodologias analíticas para a identificação e quantificação destes em alimentos industrializados. O objetivo deste trabalho foi a avaliação das condições experimentais e voltamétricas para o desenvolvimento de uma metodologia eletroanalítica para a identificação e quantificação de Níquel em alimentos industrializados empregando-se como sensor o eletrodo sólido de amálgama de prata (AgSAE) aliado à técnica analítica de voltametria de onda quadrada (SWV) com etapas de redissolução adsortiva. Foram avaliadas as respostas analíticas em meio de $0,1 \mathrm{~mol} \mathrm{~L}^{-1}$ de solução tampão amônio em diferentes valores de $\mathrm{pH}(\mathrm{pH}$ $8,0$ a 10,0$)$, seguidos da avaliação da influência da aplicação de potenciais $\left(E_{\text {acc }}\right)$ e tempos para preconcentração adsortiva $\left(\mathrm{t}_{\mathrm{acc}}\right) \mathrm{de} \mathrm{Ni}^{+2}$ sobre o AgSAE, ambos com varredura de potencial elétrico no intervalo de 0,00 a $-1,70 \mathrm{~V}$. Os parâmetros da SWV também foram avaliados, onde frequência de aplicação dos pulsos de potencial $(f)$ foi variada de 10 a $300 \mathrm{~s}^{-1}$, a amplitude de pulsos $(a)$ de -5 a $-50 \mathrm{mV}$ e o incremento de varredura de potenciais $(\Delta E s)$ de -2 a $-10 \mathrm{mV}$. A adição do agente complexante dimetilglioxima (DMG) também foi analisada, observando-se uma intensa melhoria na sensibilidade e seletividade, caracterizada pela adsorção acumulativa do dimetilglioximato de níquel na superfície do AgSAE, seguida por varreduras catódicas de potenciais. Assim os parâmetros experimentais e voltamétricos otimizados foram: $0,10 \mathrm{~mol} \mathrm{~L}^{-1}$ de solução tampão amônio $\mathrm{pH} 9,00$, $90 \mu \mathrm{mol} \mathrm{L}{ }^{-1}$ de DMG, $\mathrm{E}_{\text {acc }}$ de $-0,857 \mathrm{~V}$ por $\mathrm{t}_{\mathrm{acc}} 30$ segundos, $f=100 \mathrm{~s}^{-1}, \Delta \mathrm{Es}=-4$ $\mathrm{mV}$ e $a=-25 \mathrm{mV}$. Os parâmetros analíticos (precisão, exatidão, faixa dinâmica linear, limites de detecção, limites de quantificação e eficiência de recuperação) foram avaliados em eletrólito puro, e os resultados obtidos apresentaram valores comparáveis àqueles obtidos utilizando-se técnicas convencionais como a espectroscopia de massa com plasma acoplado indutivamente.
\end{abstract}




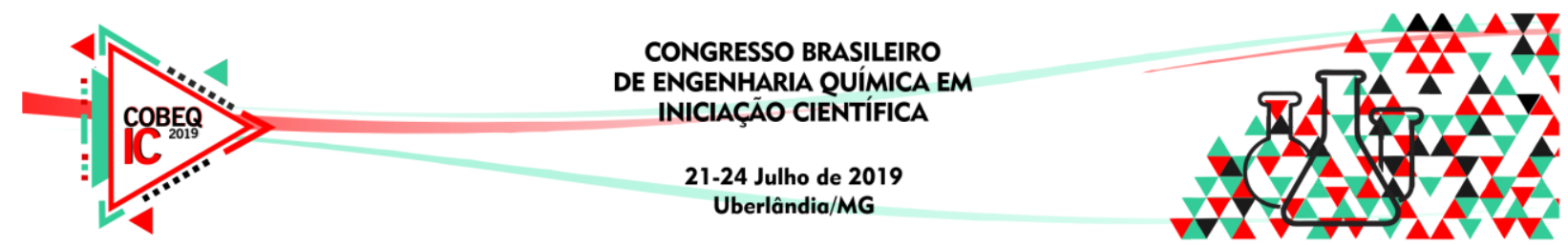

\section{INTRODUÇÃO}

O níquel é um dos cinco elementos mais abundantes da crosta terrestre e constitui aproximadamente $0,008 \%$. Encontrado na forma inorgânica como hidróxidos, sulfatos, cloretos e nitratos (compostos solúveis) e como óxidos, sulfetos e subsulfetos (compostos insolúveis). Na forma orgânica é encontrado como tetracarbonila de níquel, um composto altamente tóxico (Azevedo e Chasin, 2003).

De acordo com Sharma (2013), alguns alimentos apresentam níquel em sua composição, dentre eles estão: grãos integrais (trigo, centeio, aveia, trigo mourisco), cacau, produtos de soja, feijão vermelho, ervilhas, lentilhas, amendoim, grão de bico, frutas secas. Também pode estar presente na composição de alimentos industrializados tais como chocolates, chás, gelatinas, fermento em pó, alimentos enlatados, bebidas, alcaçuz forte e alguns suplementos vitamínicos.

A exposição a altas concentrações de níquel pode causar efeitos tóxicos, principalmente nas pessoas que possuem sensibilidade ou alergia a este metal. Entre 7,0 e 10,0 \% dos casos de dermatite alérgica são relacionados ao consumo ou contato com o níquel, onde os principais sintomas de contaminação são dermatite em pápulas vesicular eritematosa crônica e urticária CARRAPATOSO et al. (2004).Além disso vale ressaltar que a ingestão de níquel, em concentrações acima de $500 \mu \mathrm{g} \mathrm{dia}{ }^{-1}$ ou cerca de $8 \mu \mathrm{g} \mathrm{dia}^{-1}$ por peso corpóreo, pode agravar o eczema cutâneo em indivíduos com sensibilidade a níquel Authority, (2006).

Desta forma é de suma importância a utilização de técnicas analíticas sensíveis para a identificação e quantificação de níquel em diferentes níveis de concentrações. Assim, o objetivo deste trabalho foi à otimização experimental e voltamétrica para o desenvolvimento de uma metodologia eletroanalítica para determinação de Níquel em alimentos, empregandose eletrodo sólido de amálgama de prata (p-AgSAE) aliado à técnica de voltametria de onda quadrada (SWV).

\section{MATERIAIS E MÉTODOS}

Todos os experimentos eletroanalíticos foram realizados utilizando-se um potenciostato/galvanostato Metrohm-Ecochimie modelo microAutolab III controlado por uma interface com o software NOVA ${ }^{\circledR}$ 2.1.2, também da Metrohm-Ecochimie,. Uma célula eletroquímica contendo o eletrodo de trabalho (AgSAE), o eletrodo auxiliar (fio de platina), e eletrodo de referência $\left(\mathrm{Ag} / \mathrm{AgCl} 3,00 \mathrm{~mol} \mathrm{~L}^{-1}\right)$ juntamente com o eletrólito suporte (solução tampão amônio) foi empregada. Os eletrodos foram conectados ao potenciostato/galvanostato para a realização das medidas de corrente após a do potencial elétrico produzidos à partir da aplicação da SWV.

O pH da solução tampão amônio foi variado de 8,00 a 10,00 e experimentos com adições de dimetilglioxima (DMG), um agente complexante foram realizados. Também foram avaliados a influência da aplicação de potenciais e tempos para preconcentração adsortiva de $\mathrm{Ni}^{+2}$ sobre o AgSAE, seguidos por varredura de potenciais no intervalo de potencial de $0,00 \mathrm{a}$ $-1,70 \mathrm{~V}$, durante tempos de 0 a 90 segundos, empregando a técnica SWV. Os valores para frequência $(f)$, amplitude $(a)$ e incremento $\left(\Delta \mathrm{E}_{\mathrm{S}}\right)$, parâmetros da SWV que exercem grande 


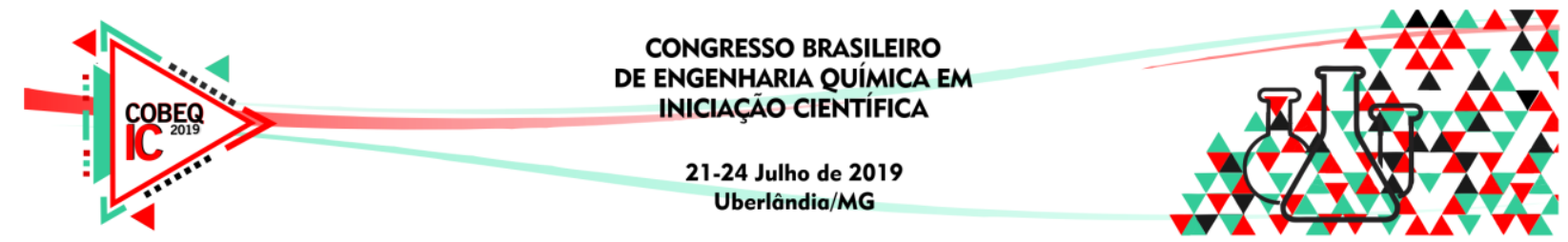

influência no perfil voltamétrico para a redução de $\mathrm{Ni}^{+2}$, definindo-se assim, a sensibilidade e seletividade da metodologia. Cada parâmetro foi individualmente avaliado, mantendo-se fixo dois dos parâmetros variando-se o outro. Assim, os valores de $f$ foram variados de 10 a 300 $\mathrm{s}^{-1}, a$ de $-5 \mathrm{a}-50 \mathrm{mV}$ e $\Delta \mathrm{E}_{\mathrm{s}}$ de $-2 \mathrm{a}-10 \mathrm{mV}$ e ajustados durante o experimentos.

Após a otimização dos parâmetros experimentais e voltamétricos, curvas analíticas (ou curvas de calibração) foram construídas utilizando-se o método de adição padrão Skoog et al. (2005). O desvio padrão $\left(S_{\mathrm{b}}\right)$ da média dos interceptos no eixo y das três curvas construídas e a inclinação das curvas analíticas (s) foram empregados para o cálculo dos limites de detecção (LOD) e de quantificação (LOQ), de acordo com as Equações 1 e 2, respectivamente Harris,(2005).

$$
\begin{aligned}
& \mathrm{LOD}=3 \mathrm{~S}_{\mathrm{b}} / \mathrm{s} \\
& \mathrm{LOQ}=10 \mathrm{~S}_{\mathrm{b}} / \mathrm{s}
\end{aligned}
$$

\section{RESULTADOS E DISCUSSÃO}

Para avaliação dos sinais voltamétricos do $\mathrm{Ni}^{+2}$ sobre AgSAE, aplicou-se potencial de preconcentração em $-0,94 \mathrm{~V}$ por 30 segundos, seguidos por experimentos de SWV empregando-se $f=100 \mathrm{~s}^{-1}, a=-50 \mathrm{mV}$ e $\Delta \mathrm{E}_{\mathrm{s}}=-2 \mathrm{mV}$, onde observou-se que o $\mathrm{Ni}^{+2}$ apresentou um pico de redução bem definido com um máximo de intensidade em aproximadamente $-0,75 \mathrm{~V}$ com o aumento da intensidade de corrente proporcionalmente com o aumento da concentração de $\mathrm{Ni}^{+2}$ em solução, como pode ser observado na Figura 1 (a).

Com a adição de DMG ao meio pode-se observar que o pico voltamétrico tornou-se mais estreito, proporcionando melhor seletividade analítica, com redução da variação de largura de meia-altura de $135 \mathrm{mV}$ para $34 \mathrm{mV}$, considerando-se os voltamogramas sem adição de DMG e com adição de DMG à solução, como mostrado na Figura 1 (b).

Figura 1- (a)Voltamograma de onda quadrada para a redução de diferentes concentrações de $\mathrm{Ni}^{+2}$ em meio de 0,10 mol L ${ }^{-1}$ de solução tampão amônio $\mathrm{pH}$ 8,50 sobre AgSAE, aplicando-se potencial de preconcentração de $-0,94 \mathrm{~V}$ por 30 segundos, $\operatorname{com} f=100 \mathrm{~s}^{-1}, \Delta \mathrm{E}_{\mathrm{s}}=-2 \mathrm{mV}$ e $a=-50 \mathrm{mV}$. (b) Voltamograma de onda quadrada para a redução de $\mathrm{Ni}^{+2}$ e $50 \mu \mathrm{mol} \mathrm{L}^{-1} \mathrm{de}$ DMG sobre AgSAE em meio 0,10 mol L ${ }^{-1}$ de solução tampão amônio pH 9,00, com $f=100$ $\mathrm{s}^{-1}, \Delta \mathrm{E}_{\mathrm{s}}=-2 \mathrm{mV}$ e $a=-50 \mathrm{mV}$.
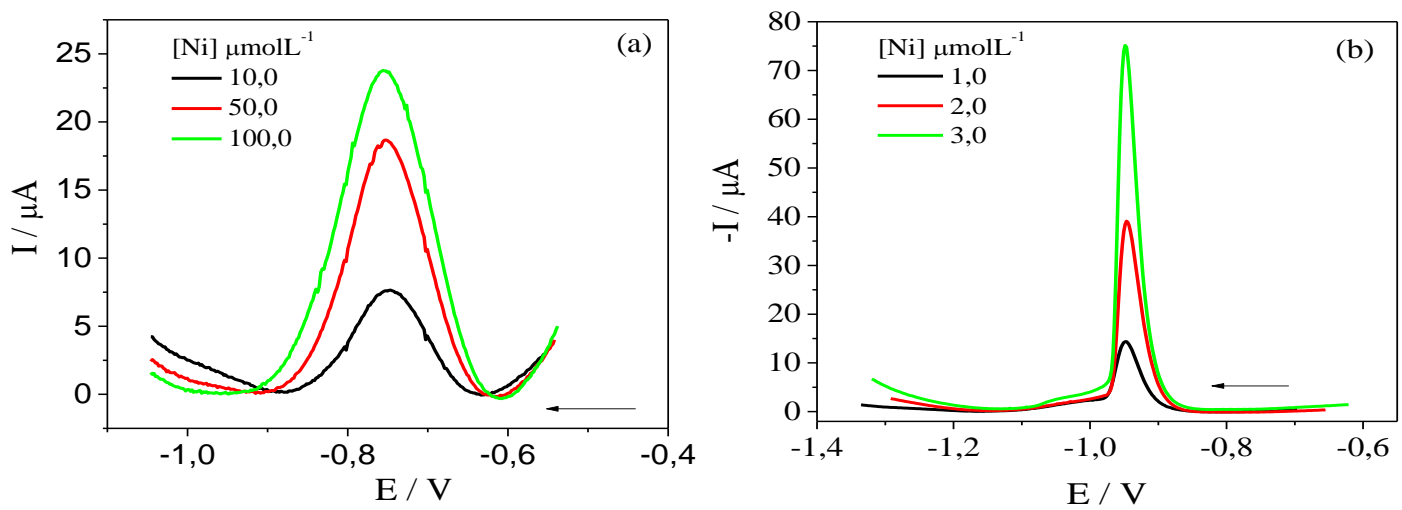


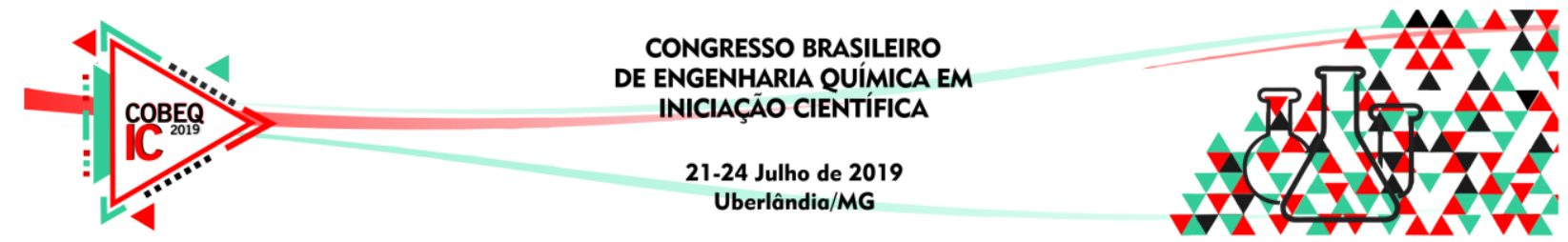

A intensidade de sinal de corrente em 1(b) aumentou consideravelmente, pois o $\mathrm{Ni}^{+2}$ sofreu reação de complexação com DMG, e posteriormente o complexo Ni(DMG) ${ }_{2}^{+2}$ formado sofreu reação de redução envolvendo maior número de elétrons que a simples redução de $\mathrm{Ni}^{+2}$, como mostrado no esquema da Figura 2.

Figura 2- Reação dos íons $\mathrm{Ni}^{+2}$ com DMG.

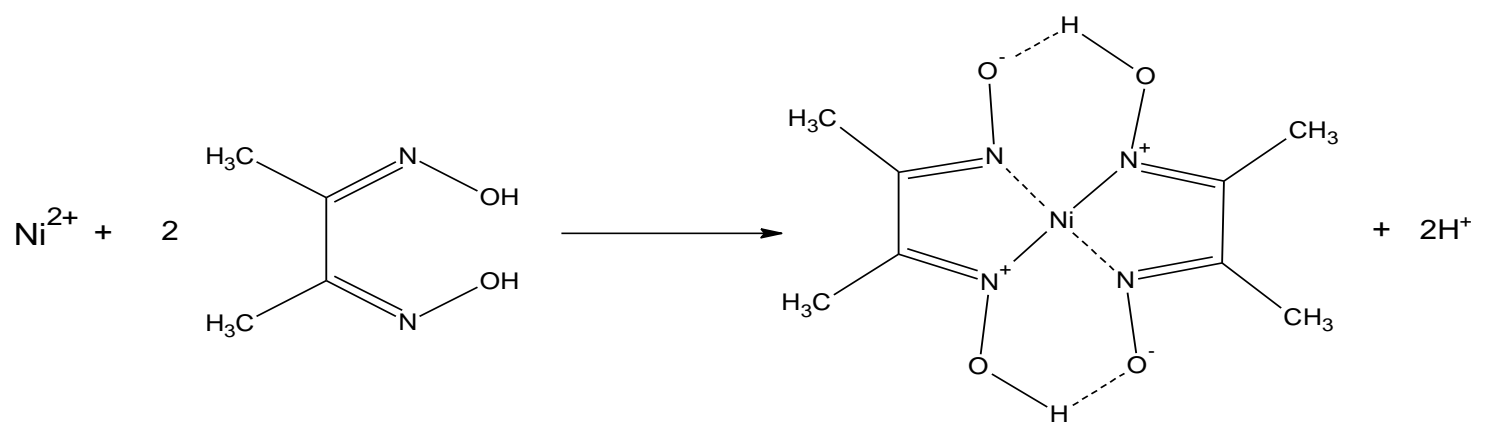

O efeito das proporções molares entre de $\mathrm{Ni}^{+2}$ e DMG foi analisado e os resultados são apresentados na Figura 3. A razão de concentração de ambas as espécies influenciou de forma significativa o perfil voltamétrico. Proporções de complexante menores que de $\mathrm{Ni}^{+2}$, apresentaram uma perda de definição do pico voltamétrico, além disso houve um aumento na largura de meia-altura $\left(\Delta \mathrm{E}_{\mathrm{p} 1 / 2}\right)$, diminuindo a sensibilidade e seletividade analítica. Entretanto, maior proporção de complexante que de $\mathrm{Ni}^{+2}$, obteve-se melhor definição do pico voltamétrico, com aumento nas intensidades de corrente $\left(\mathrm{I}_{\mathrm{p}}\right)$, indicando que a concentração de complexante deve ser maior que a de $\mathrm{Ni}^{+2}$.

Figura 3- Voltamogramas de onda quadrada para a redução de $\mathrm{Ni}^{+2}$ com DMG sobre AgSAE em meio de 0,10 mol L $^{-1}$ de tampão amônio $\mathrm{pH} 9,00$, com $\mathrm{E}_{\mathrm{acc}}=-0,857 \mathrm{~V}, \mathrm{t}_{\mathrm{acc}}=30 \mathrm{~s}, f=100$ $\mathrm{s}^{-1}, \Delta \mathrm{E}_{\mathrm{s}}=-4 \mathrm{Mv}, a=-25 \mathrm{mV}$ considerando-se diferentes proporções entre as razões molares de $\mathrm{Ni}^{+2}$ e DMG.

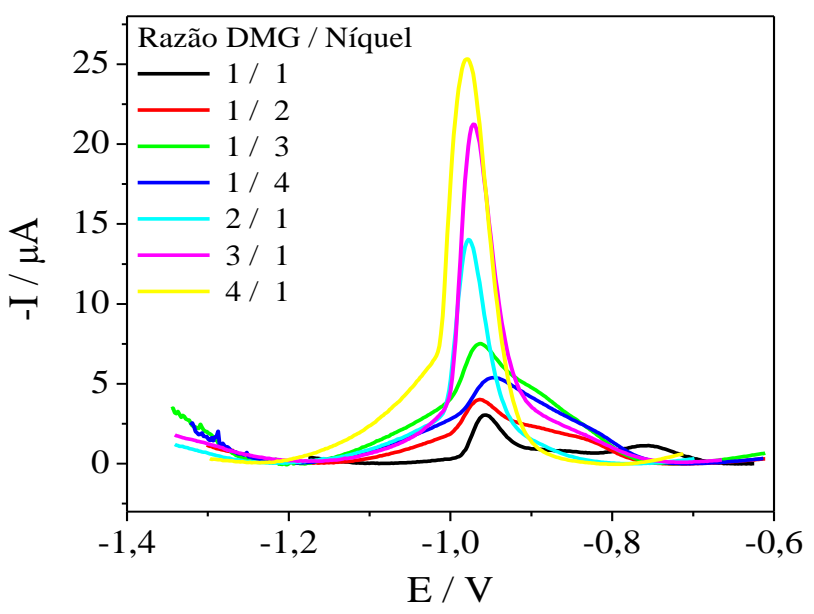

Estes dados são concordantes com a estequiometria de formação do complexo $\left[\mathrm{Ni}(\mathrm{DMG})_{2}{ }^{+2}\right]$, mostrada na Figura 1 . Assim, excesso de DMG foi adicionado à célula eletroquímica para garantir completa e rápida complexação, de maneira que a proporção 4:1 


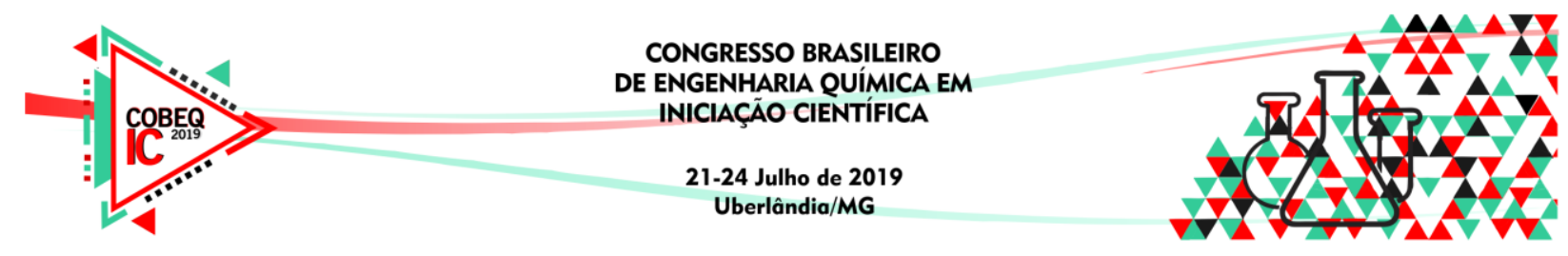

(DMG: $\mathrm{Ni}^{+2}$ ) foi selecionada para as etapas posteriores, pois permitiu a obtenção de maiores intensidades de corrente.

Após todos os parâmetros experimentais e voltamétricos estarem otimizados a metodologia foi empregada para a construção de curvas de calibração utilizando-se adições sucessivas de solução padrão de $\mathrm{Ni}^{+2}$ a uma célula eletroquímica contendo o eletrólito de suporte e $90 \mu \mathrm{mol} \mathrm{L}{ }^{-1}$ de DMG.

Os voltamogramas resultantes de adições sucessivas de $\mathrm{Ni}^{+2}$ no intervalo de concentração de $1,00 \times 10^{-8} \mathrm{~mol} \mathrm{~L} \mathrm{~L}^{-1}$ a $1,50 \times 10^{-7} \mathrm{~mol} \mathrm{~L}^{-1}$ a partir de uma solução estoque $1,00 \times 10^{-5} \mathrm{~mol} \mathrm{~L}^{-1}$ de $\mathrm{Ni}^{+2}$ estão representados na Figura 4.

Figura 4- (a) Voltamogramas de onda quadrada para a redução de $\mathrm{Ni}^{+2}$ com $90,0 \mu \mathrm{mol} \mathrm{L}^{-1}$ de DMG sobre AgSAE em meio de 0,10 mol L $^{-1}$ de solução tampão amônio $\mathrm{pH} 9,00$, com $\mathrm{E}_{\mathrm{acc}}=$ $-0,857 \mathrm{~V}, \mathrm{t}_{\mathrm{acc}}=30 \mathrm{~s}, f=100 \mathrm{~s}^{-1}, a=-25 \mathrm{mV}$ e $\Delta \mathrm{E}_{\mathrm{s}}=-4 \mathrm{mV}$ considerando-se diferentes de $\mathrm{Ni}^{+2}$. (b) Relações de $\mathrm{I}_{\mathrm{p}}$ versus $\left[\mathrm{Ni}^{+2}\right]$ obtida a partir dos voltamogramas apresentados em (a).
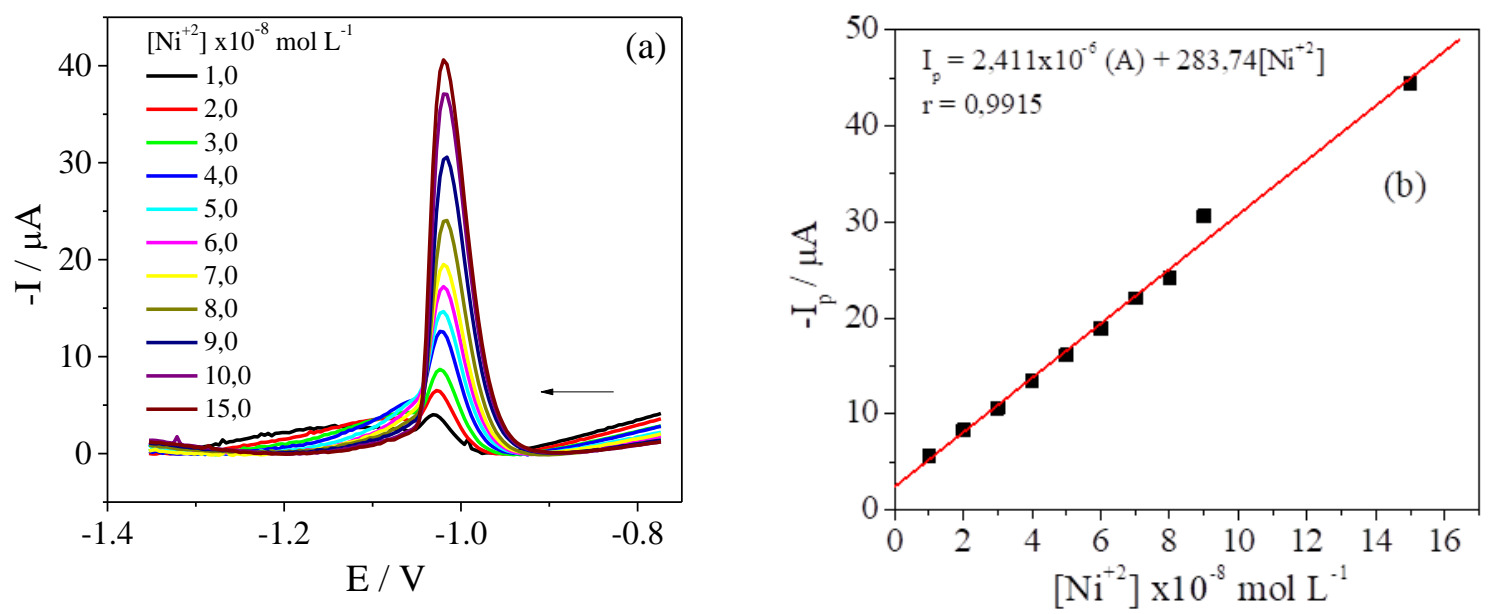

Observou-se que as correntes de pico aumentaram proporcionalmente com as adições de $\mathrm{Ni}^{+2}$, sem variações significativas no potencial de pico e largura de meia-altura, apresentou aumento linear de acordo com a Equação 3, que representa a regressão linear obtida com coeficiente de correlação (r) de 0,9915.

$$
\mathrm{Ip}=2,411 \times 10^{-6}(\mathrm{~A})+283,74\left[\mathrm{Ni}^{+2}\right]
$$

A partir dos resultados encontrados da equação acima, valores de LOD e LOQ foram calculados a partir dos dados da curva de calibração média e empregando-se a Equação $1 \mathrm{e}$ Equação 2, os valores encontrados foram $1,325 \times 10^{-9} \mathrm{~mol} \mathrm{~L}^{-1} \mathrm{e} 4,416 \times 10^{-9} \mathrm{~mol} \mathrm{~L}^{-1}$ respectivamente.

Após a otimização experimental construiu-se a Tabela 1 que apresenta os parâmetros analíticos calculados com a metodologia proposta para determinação de $\mathrm{Ni}^{+2}$. Os valores apresentados indicam que a metodologia proposta é adequada para a identificação e quantificação de $\mathrm{Ni}^{+2}$ em eletrólito puro, e poderá ser empregada em amostras complexas, tais como alimentos industrializados. 


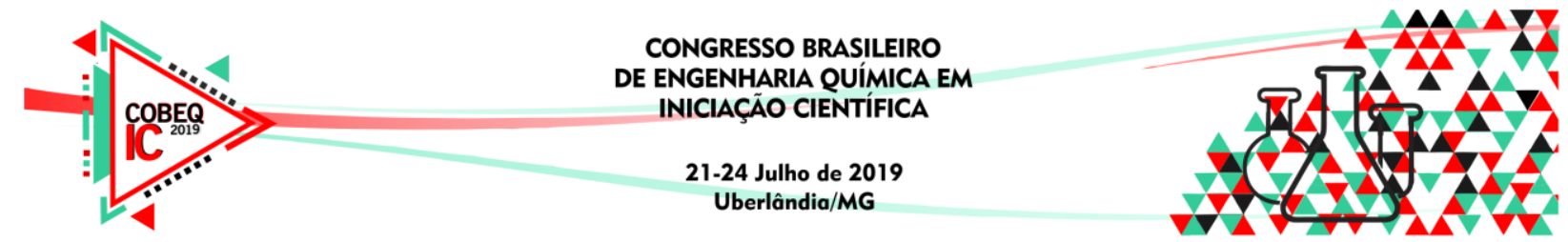

Tabela 1- Parâmetros analíticos calculados para a metodologia proposta para a determinação de $\mathrm{Ni}^{+2}$ sobre AgSAE em meio de $0,1 \mathrm{~mol} \mathrm{~L}^{-1}$ de solução tampão amônio pH 9,00 e SWV com acumulação em $\mathrm{E}_{\mathrm{acc}}=-0,857 \mathrm{~V}, \mathrm{t}_{\mathrm{acc}}=30 \mathrm{~s}, f=100 \mathrm{~s}^{-1}, a=-25 \mathrm{mV}$ e $\Delta \mathrm{E}_{\mathrm{s}}=-4 \mathrm{mV}$.

\begin{tabular}{|c|c|}
\hline Parâmetros & Valor \\
\hline Faixa de Concentração (x10 $\left.{ }^{-8} \mathrm{~mol} \mathrm{~L}^{-1}\right)$ & $1,00-15,00$ \\
\hline Intercepto (A) & $2,411 \times 10^{-6}$ \\
\hline $\mathrm{s}(\mathrm{A} / \mathrm{mol} \mathrm{L}$ & \\
\hline $\mathrm{r}$ & 283,74 \\
\hline $\mathrm{S}_{\mathrm{b}}(\mathrm{A})$ & 0,9915 \\
\hline LOD (mol L-1) & $1,253 \times 10^{-7}$ \\
\hline LOQ (mol L $)$ & $1,325 \times 10^{-9}\left(1,325 \mathrm{nmol} \mathrm{L}^{-1}\right)$ \\
\hline Repetibilidade (\%) & $4,416 \times 10^{-9}\left(4,416 \mathrm{nmol} \mathrm{L}^{-1}\right)$ \\
\hline Reprodutibilidade (\%) & 3,13 \\
\hline Recuperação (\%) & 3,04 \\
\hline
\end{tabular}

\section{CONCLUSÃO}

A utilização de AgSAE aliada a SWV com redissolução adsortiva permitiu desenvolver a metodologia eletroanalítica para identificação de níquel, sendo esta adequada em termos de precisão, exatidão, faixa dinâmica linear, eficiência de recuperação, sensibilidade e seletividade. Estes parâmetros foram avaliados após a otimização experimental e voltamétrica, indicando que a metodologia proposta apresentou parâmetros comparáveis empregando-se ICP-MS, a técnica espectroscópica mais sensível e seletiva.

\section{REFERÊNCIAS}

AUTHORITY, E. F. S. Tolerable upper intake levels for vitamins and minerals, 2006.

AZEVEDO, Fausto Antônio de; CHASIN, Alice A. da Matta. Metais - Gerenciamento da Toxicidade. Atheneu, 2003. 554 p.

CARRAPATOSO, I. et al. Dermatite endógena induzida pela ingestão de níquel . A propósito de dois casos clínicos. p. 261-270, 2004.

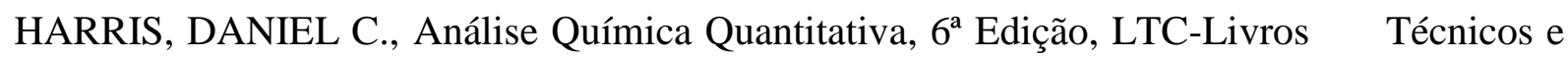
Científicos Editora S.A., Rio de Janeiro-RJ, 2005.

SKOOG, D. a. et al. Fundamentos de Química Analítica. Fundamentos de Química Analítica, v. 58, p. 374, 2005.

SHARMA, A. Low nickel diet in dermatology. Indian Journal of Dermatology, 2013. 